\title{
BOUNDING DATA WITH A PIECEWISE LINEAR BAND*
}

\author{
ALLAN R. WILLMS
}

\begin{abstract}
This paper presents an algorithm for finding a piecewise linear band which bounds given two-dimensional data. The band is of constant height, and the distance between adjacent slope discontinuities in opposite directions is restricted by two user-specified parameters.
\end{abstract}

Key words. bounding data, piecewise linear bounds, parameter estimation

AMS subject classifications. 65D10, 65G40

DOI. $10.1137 / 080732316$

1. Introduction. A time series data set $S=\left\{\left(t_{i}, y_{i}\right)\right\}_{i=1}^{n}$ is generally subject to measurement errors for the $y$-values. Often a simplified representation of these data is constructed: either a parameterized curve with parameters chosen to "best fit" the data or a smooth spline. Such representations yield curves which pass through the "middle" of the data and typically also provide some overall measure of the distance from the fitted curve to the data. However, in some applications a representation which specifically bounds the data is desirable. Particularly, parameter estimation methods for ordinary differential equation (ODE) models using interval analysis [1, $2,5,6,7]$ or monotonic discretizations [8], and various methods for fitting curves to data $[3,4]$, typically start with the assumption that the observed data lie within some known error bound. If the source of the noise in the data is simply from within the measurement instrument, then it is possible that it can be quantified and the data point represented as $y_{i} \pm \epsilon$ with $\epsilon$ reasonably known. However, if, as is usually the case, the noise is externally sourced, it may not be easily quantifiable and reasonable values for $\epsilon$ must somehow be inferred from the data.

Good bounds for the data are an essential starting point for parameter identification techniques based on interval analysis [7]. A parameterized function (typically the solution of a system of ODEs) is assumed to represent the data. With these techniques one proves that provided the parameter values lie within some specified box in parameter space, then the function defined by these parameters yields values (intervals) which are within, intersect, or do not intersect prespecified intervals around the data. The determination of the boxes in parameter space is driven by the given data intervals; the smaller these intervals are, the more tightly one can constrain the parameter boxes which intersect the data intervals. On the other hand, if the specified intervals are too small compared to the actual noise level in the data, then these techniques will reach the conclusion that no parameter values will allow the function to be consistent with the data. It is therefore necessary to provide intervals around the data which are large enough to account for noise but small enough to not lose the signal present in the data.

Here we introduce a simple algorithm which constructs a piecewise linear band (two piecewise linear curves differing by a constant vertical offset) which tightly bounds the given data set $S$. The algorithm is controlled by two parameters $t_{\min }$

\footnotetext{
${ }^{*}$ Received by the editors August 6, 2008; accepted for publication (in revised form) February 5, 2009; published electronically May 28, 2009.

http://www.siam.org/journals/sisc/31-3/73231.html

$\dagger$ Department of Mathematics and Statistics, University of Guelph, Guelph, ON, N1G 2W1, Canada (awillms@uoguelph.ca).
} 
and $M$ which regulate, as described below, the distance between points where the band changes slope. The result is a simple characterization of the uncertainty in the data and may be used as input to the various parameter estimation methods mentioned above.

2. The algorithm. The way in which the algorithm constructs the band around the data can be compared to dropping a stretchable but stiff cord onto the data points from above (and simultaneously one from below). When the cord hits a data point, that point becomes fixed and the cord continues to drop elsewhere.

The piecewise linear band that will be constructed around the data set $S=$ $\left\{\left(t_{i}, y_{i}\right)\right\}_{i=1}^{n}\left(t_{i}\right.$ increasing $)$ is represented by an index set $J=\left\{j_{k}\right\}_{k=1}^{N} \subseteq\{1, \ldots, n\}$ ( $j_{k}$ increasing), a height $h \geq 0$, and a placement function $\sigma: J \rightarrow\{0,1\}$. Each index $j \in J$ selects a time point $t_{j}$ of the original data set at which the band changes slope; this point is called a control point. Each control point has an associated range $\left[z_{j}, z_{j}+h\right]$, where

$$
z_{j}=y_{j}-\sigma(j) h
$$

that defines the band at that point. The function $\sigma$ determines whether the data value $y_{j}$ touches the lower edge of the band $(\sigma(j)=0)$ or the upper edge $(\sigma(j)=1)$. In the former case the control point is called a "lower control point" and in the latter case an "upper control point." For lower control points, the lower boundary is the "fixed end" and the upper boundary is the "free end," and vice versa for upper control points. Between the $N$ control points the band's upper and lower boundaries are linear functions, and beyond the first and last control points, the band is constant. Thus the lower boundary of the band is given by

$$
\underline{B}(t)= \begin{cases}z_{j_{1}} & \text { if } t \leq t_{j_{1}}, \\ z_{j_{k-1}}+\frac{z_{j_{k}}-z_{j_{k-1}}}{t_{j_{k}}-t_{j_{k-1}}}\left(t-t_{j_{k-1}}\right) & \text { if } t_{j_{k-1}}<t \leq t_{j_{k}}, \quad 1<k \leq N, \\ z_{j_{N}} & \text { if } t_{j_{N}}<t\end{cases}
$$

and the upper boundary is simply $\bar{B}(t)=\underline{B}(t)+h$.

The placement of control points is constrained by the parameters $t_{\min }$, a nonnegative real, and $M$, a nonnegative integer. Adjacent control points $t_{j_{k-1}}$ and $t_{j_{k}}$ are considered close if $t_{j_{k}}-t_{j_{k-1}}<t_{\text {min }}$. The algorithm permits no more than $M$ control points of opposite type (lower or upper) to be close. Smaller values of $t_{\text {min }}$ and larger values of $M$ allow the band to be narrower at the expense of more changes in slope. In the one extreme, if $M$ is set to the number of data points or $t_{\min }$ is set below the smallest distance between any two $t_{i}$ of the data set, then the algorithm will return a band with height zero; that is, every data point will be a control point. At the other extreme, if $M=0$ and $t_{\min } \geq t_{n}-t_{1}$, then the algorithm will return a constant band of height equal to the distance between the maximum and minimum values of $y$, that is, a single control point located where $y$ is either maximal or minimal.

The algorithm begins by placing control points where the maximum and minimum values of $y$ occur, with the former being an upper control point and the latter a lower one. The height $h$ of the band $B(t)$ is set to the difference between the maximum and the minimum $y$ values. The algorithm reduces the height of the band by simultaneously moving all the control point free ends toward the fixed ends at an equal rate until one of the boundaries of the band touches a data value $y_{i}$ for which $t_{i}$ is not yet a control point. This point is then added to the control point list, and 
the algorithm continues. As control points are added, the distances between them are calculated and the count of close control points of opposite type is adjusted as necessary. When a new control point is to be added which would increase the number of close control points of opposite type beyond the maximum number allowed, the algorithm terminates. The algorithm will also terminate if the height of the band reaches zero.

The following pseudocode describes an implementation for this algorithm. An array of control points is maintained that is sorted in increasing $t_{j}$ values. Each iteration reduces the height of the band and adds a new control point to the list. In each iteration, for each control point with an unknown reduction level for each data point between this control point and the next (for the first control point, the data points to the left of it are also tested), the free ends of the control points are temporarily moved so that the boundary of the band passes through the data point. The location of the free point determines the possible reduction in the height of the band segment to the right of the control point. (Note that if the two control points are of the same type, then moving the free ends moves only one boundary of the band segment while if the control points are of opposite type, both boundaries move and the possible reductions from both situations are computed.) The smallest of these possible reductions is selected and becomes the reduction level for this control point. The smallest reduction level of all control points is then subtracted from the height of the band and all control point reduction levels. The reduction level for the control point which had the smallest reduction level is marked as unknown, and the constraining data point becomes a new control point with unknown reduction level. C code can be obtained from http://www.uoguelph.ca/ ${ }^{\sim}$ awillms/linenvelope/.

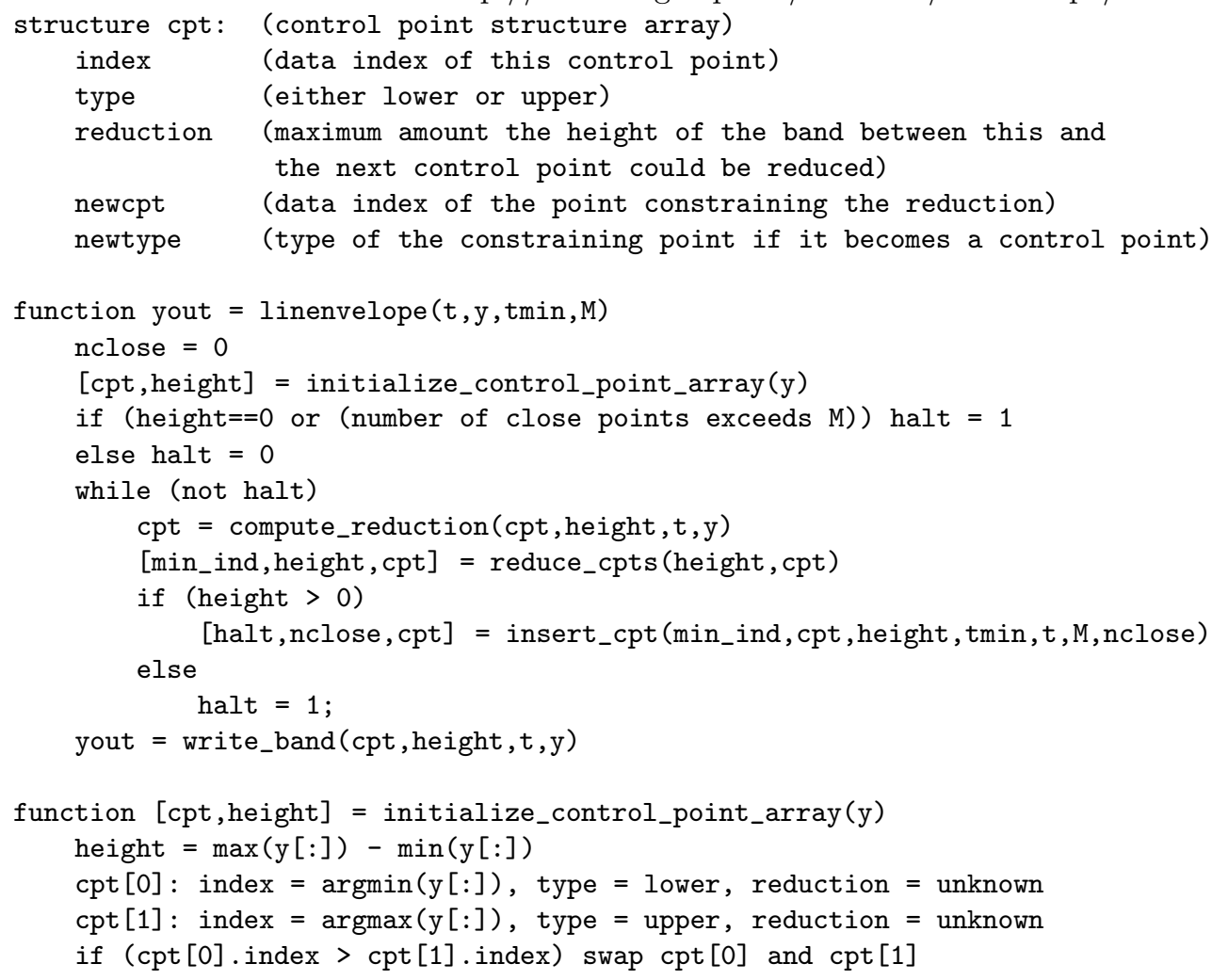




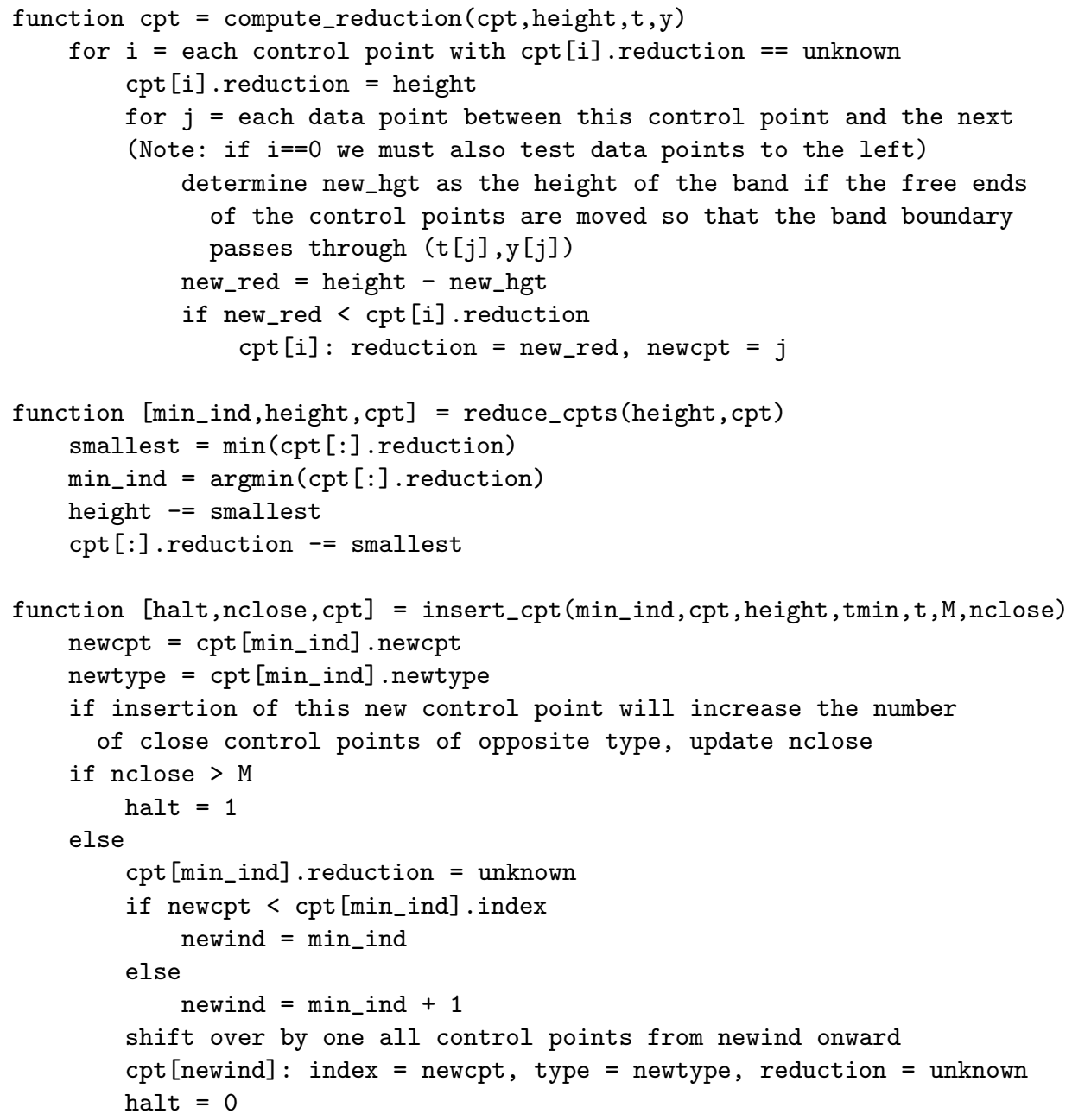

For height reduction calculation purposes, the data points associated with each control point are those between it and the next control point to the right, except for the first control point which also has the data points to the left associated to it. On each iteration, only two control points will have a reduction value of unknown when the function compute_reduction is called: the most recently added control point and the one to its left (or right if the newest point is the lowest $t_{j}$ value). To compute the reduction values for the data points associated to these two control points requires eight arithmetic operations per data point. As control points are added, the average number of data points associated to each control point decreases. With the assumptions that when $k$ control points are present, the average number of data points associated to each control point is $n / k$, and that half the time adjacent control points are of the same type so that just one boundary moves, the total number of arithmetic operations required to select $N$ control points for $n$ data points is approximately bounded by $24 n \ln (N)$. Additionally, the number of arithmetic operations required by the function reduce_cpts is bounded by $N^{2} / 2$.

3. Examples. The algorithm's ability is illustrated in the following examples. The first is simply a sine wave:

$$
y_{i}=10 \sin \left(t_{i}\right)+\epsilon\left(t_{i}\right), \quad t_{i}=(i-1) / 10, \quad 1 \leq i \leq 100=n,
$$


A
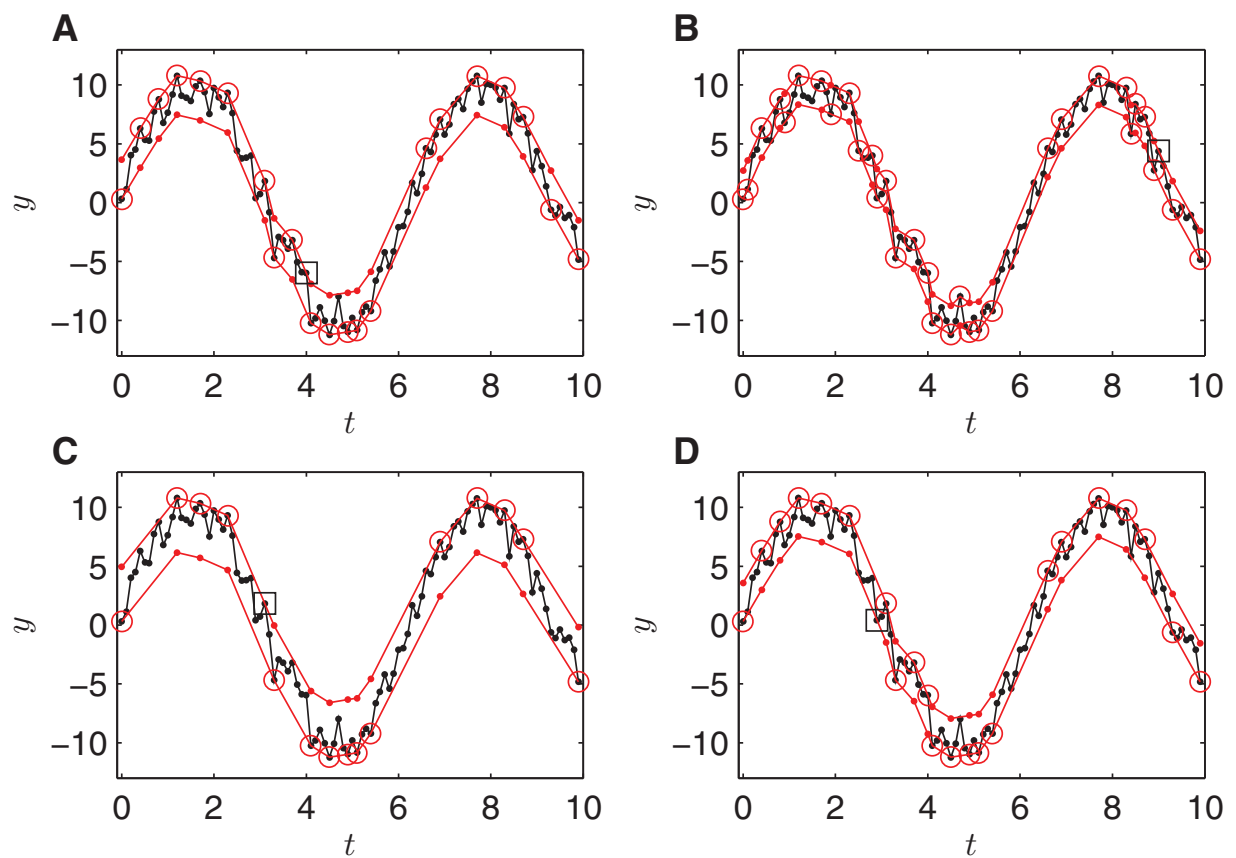

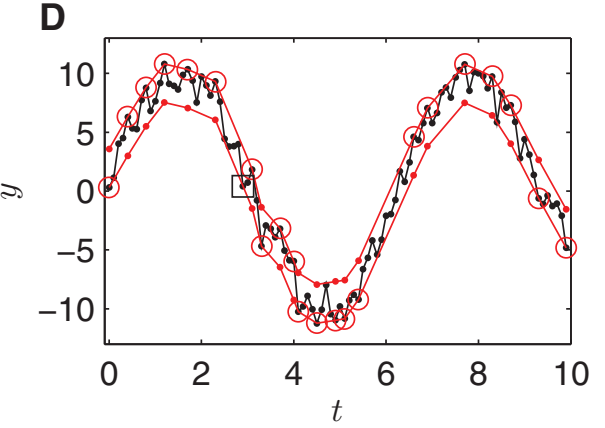

FIG. 3.1. Sine wave data (see (3.1)) with bands fitted using various $t_{\min }$ and $M$ values. Control points are marked with a circle at the fixed end and a dot at the free end. The data point marked with a square is the one that caused the algorithm to stop since it would increase the number of close control points of opposite type beyond the maximum number (M) allowed. (A) $t_{\min }=0.15$, $M=0$; height is $3.34,21$ control points; (B) $t_{\min }=0.15, M=5$; height is $2.46,32$ control points; (C) $t_{\min }=0.25, M=0$; height is $4.64,15$ control points; (D) $t_{\min }=10.0, M=7$; height is 3.29 , 22 control points.

where $\epsilon$ is noise with normal distribution, mean zero, and variance 1.0. A typical instance of this data is shown in Figure 3.1. The plots in this figure show various fitted bands using different values of $t_{\min }$ and $M$. In general, lowering $t_{\min }$ or raising $M$ allows more control points and a tighter but less smooth band around the data. In Figure 3.1(A) and (B), $t_{\min }$ is set to 0.15 (between one and two times the data sampling interval) so that opposite type control points will be considered close only if they are on neighboring data points. In Figure 3.1(A), $M=0$ and the fitted band has a height of 3.34, while in Figure 3.1(B), the maximum number of close control points $M$ is increased to 5 . An additional 11 control points end up being added until the count of close points is 5 , and the height of the resulting band is 2.46. In Figure $3.1(\mathrm{C}), t_{\min }=0.25$ and $M=0$ so that to be close control points need only be within two data points of each other. The result is a wider band than Figure 3.1(A), with a height of 4.64. Finally, Figure 3.1(D) illustrates that a band similar to that in Figure 3.1 (A) can be generated by setting $t_{\min }=10.0$ and $M=7$ so that all adjacent control points of opposite type will be considered close and a total of 7 such points will be allowed. In this figure, the fixed ends alternate from one boundary to the other six times. The data point marked with a square would be the next control point, but it would increase the count of close points by two, and hence the algorithm stops.

The second example shows noisy but very dense data for the function

$$
\begin{aligned}
& y_{i}=10\left(1-e^{-t_{i} / 3}\right)^{3}\left(0.3 e^{-t_{i} / 60}+0.7 e^{-t_{i} / 15}\right)+\epsilon\left(t_{i}\right), \\
& t_{i}=(i-1) / 5,1 \leq i \leq 500=n,
\end{aligned}
$$



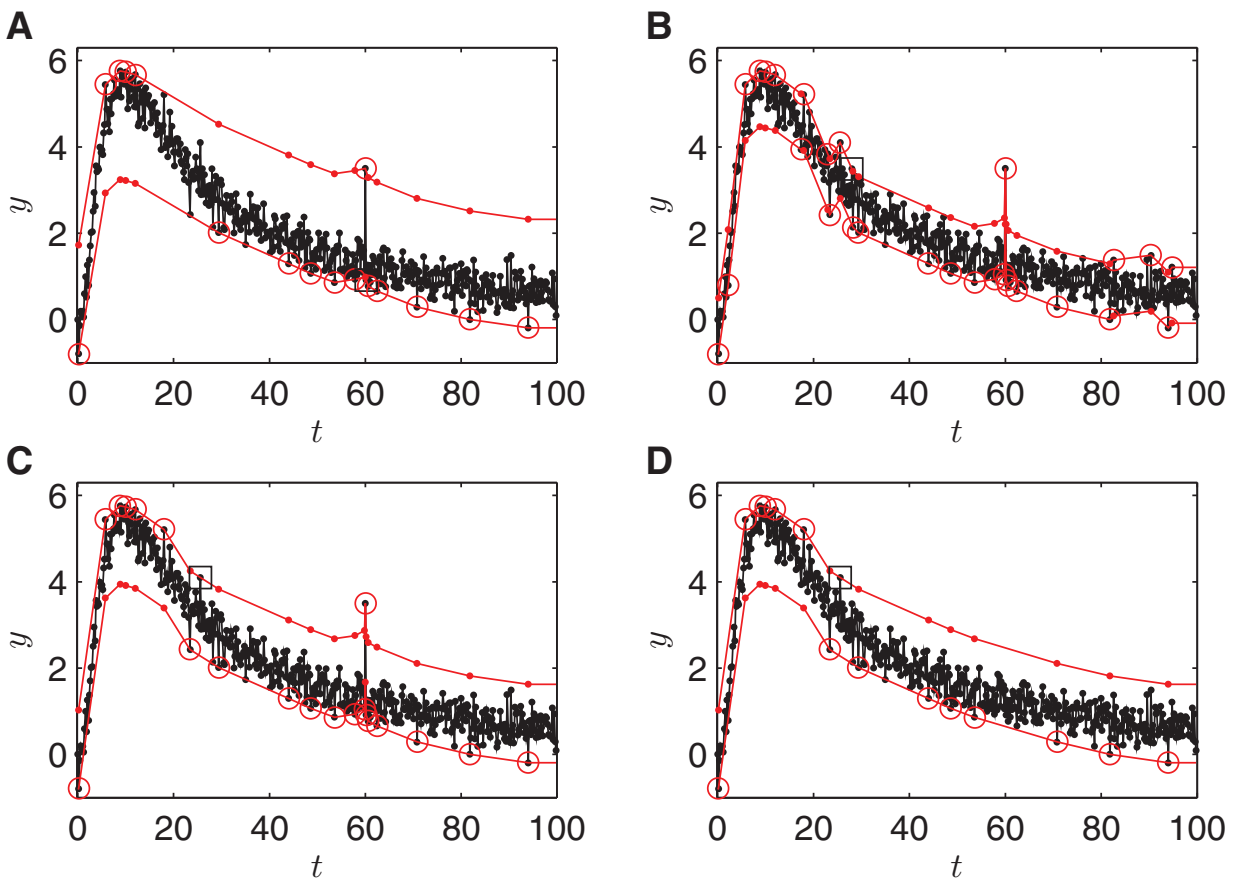

FIG. 3.2. Data for (3.2) with bands fitted using various $t_{\min }$ and $M$ values. Symbols as in Figure 3.1. (A) $t_{\min }=0.3, M=0$; height is 2.52, 17 control points; (B) $t_{\min }=0.3, M=2$; height is 1.29, 29 control points; (C) $t_{\min }=3.3, M=2$; height is 1.82, 21 control points; (D) $t_{\min }=3.3$, $M=0$, outlier removed; height is $1.82,15$ control points.

where $\epsilon$ is noise with normal distribution, mean zero, and variance 0.09. In addition, an outlier value of 3.5 is artificially placed at $t=60$. Figure 3.2 shows various band fits to one instance of these data. Since the data is sampled at an interval of 0.2 , $t_{\text {min }}=0.3$ effectively makes adjacent control points close if they are on neighboring data points. Figures $3.2(\mathrm{~A})$ and $(\mathrm{B})$ show fits with this $t_{\min }$ value and $M=0$ or $M=2$. As seen in Figure 3.2(A), the outlier at $t=60$ effectively limits the height of the fitted band to approximately the outlying distance. However, if two close control points are allowed, as in Figure 3.2(B), they are placed on either side of the outlier and the band height is reduced substantially. Figure 3.2(C) shows the result when $t_{\min }=2.3$, a much stricter definition of "close," and $M=5$. As expected, the fit is not as tight as in Figure 3.2(B). In this case the algorithm ceased due to the data point near $t=25$, which lies substantially above the control point data value near $t=23$. Figure $3.2(\mathrm{D})$ shows the fit with $t_{\min }=3.3$ as in Figure $3.2(\mathrm{C})$, but $M=0$ so that no close control points are permitted and the outlying point at $t=60$ has been removed. In this case the fit has the same width as in Figure 3.2(C) but a few less control points since the ones near the outlier are not needed. Note also that, for these fits, there happens to be no control point at the end, $t=100$, since a flat (constant) band from the last control point onward envelopes the data.

4. Discussion. The algorithm described here rapidly computes a piecewise linear band around a given time-series data set. It has the properties that all data points lie within the band and the band is the same height throughout. The tightness and smoothness of the fitted band are controlled by the two parameters $t_{\min }$ and $M$. 
The first of these defines the time interval at which opposite type control points are considered close, and $M$ limits the number of such control points permitted.

The reason for counting only control points of opposite type (lower or upper) when determining closeness is that one expects that if the data is curving, then a number of identical type control points will occur near each other along the outside of the curve. This is evident near the peak of the data in Figure 3.2 and at the peaks and troughs of the data in Figure 3.1.

Outliers in the data (if one does not specifically remove them) are effectively dealt with by allowing at least two close control points of opposite type for each outlier. The fitted band will then effectively fit the remaining data as if the outlier were not present, although some deflection of the band near the outlier may be present.

The algorithm tends to produce a band where, at a curve in the data, the convex side of the boundary tends to lie closer to the data than the concave side. This is clearly evident in both examples of the previous section. It would be desirable to have the data lie closer to the middle of the band in these cases. An additional postprocessing step could be added to the algorithm to try to shift the ranges at each control point so that the data value moves toward the center of the range. The amount of shift at each control point would depend on the nearby data (which would need to be kept within the shifting band boundaries) and the amount of shift at the neighboring control points. Consequently, this would be a global optimization problem and could not be solved simply at each control point in turn. In addition, we would not want to apply such a process at an outlying data point since this would exaggerate the outlier more. Another idea would be to try to simultaneously move the "fixed" ends of the control point interval away from the data as the free ends are moving (faster) toward it. Additional work in this direction is required.

For very noisy data, the height of the fitted band can be fairly large due to opposite acting noise at nearby data points. Another way to more tightly fit such data would be to allow the band to change height. One possible way to implement this would be to run the algorithm as given until it stops and then, fixing the height at the two control points surrounding the putative control point which stopped the algorithm, continue to reduce the intervals at the other control points until another close control point needs to be added. This process could be iteratively continued until all control point intervals have been reduced as much as possible.

\section{REFERENCES}

[1] L. JAUlin, Nonlinear bounded-error state estimation of continuous-time systems, Automatica J. IFAC, 38 (2002), pp. 1079-1082.

[2] Y. LiN AND M. A. StADTHERR, Guaranteed state and parameter estimation for nonlinear continuous-time systems with bounded-error measurements, Ind. Eng. Chem. Res., 46 (2007), pp. 7198-7207.

[3] R. K. Pearson, Fitting straight lines to poor quality $(x, y)$ data, Math. Comput. Modelling, 16 (1992), pp. 71-80.

[4] H. Piet-Lahanier ANd E. Walter, Exact recursive characterization of feasible parameter sets in the linear case, Math. Comput. Simulation, 32 (1990), pp. 495-504.

[5] W. Tucker, Z. KutAlik, And V. Moulton, Estimating parameters for generalized mass action models using constraint propagation, Math. Biosci., 208 (2007), pp. 607-620.

[6] W. Tucker and V. Moulton, Parameter reconstruction for biochemical networks using interval analysis, Reliab. Comput., 12 (2006), pp. 389-402.

[7] E. Walter AND M. KIEFFeR, Guaranteed nonlinear parameter estimation in knowledge-based models, J. Comput. Appl. Math., 199 (2007), pp. 277-285.

[8] A. R. Willms, Parameter range reduction for ODE models using cumulative backward differentiation formulas, J. Comput. Appl. Math., 203 (2007), pp. 87-102. 